\title{
Potencial de rendimiento y calidad de 13 variedades e híbridos comerciales de cítricos en condiciones del piedemonte llanero de Colombia
}

\section{Yield and quality potential of 13 commercial varieties and hybrids of citrus in the conditions of the Piedmont Plains of Colombia}
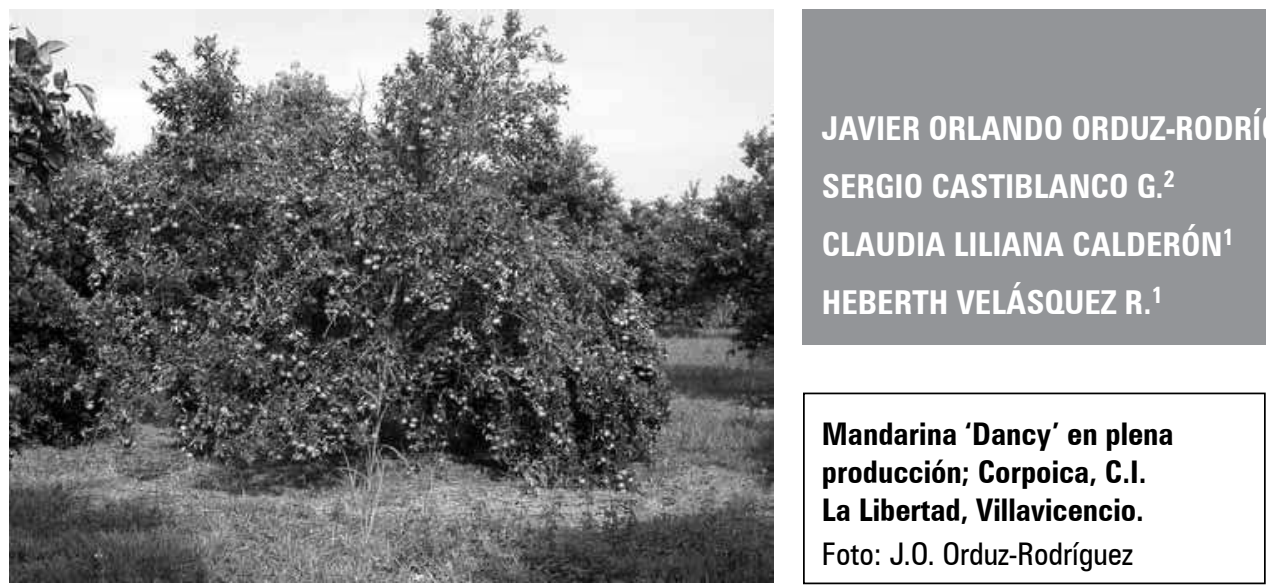

\section{RESUMEN}

Con el propósito de seleccionar cultivares de cítricos con potencial productivo y con calidad de fruta adecuada para el consumo; se evaluó el comportamiento de 13 variedades e híbridos de cítricos en condiciones del piedemonte llanero en el departamento del Meta (Colombia). Se midieron las variables de crecimiento (altura de planta, diámetro de copa), potencial productivo (rendimiento por planta y peso de fruto) y calidad del fruto (sólidos solubles totales, acidez total titulable y contenido de jugo). Los cultivares que mostraron mejor potencial productivo y de calidad del fruto fueron: mandarina 'Dancy', naranja 'Crescent Sweet', toronja 'Star Ruby', tangor 'Ellendale' y el tangelo 'Orlando'; estos pueden ser clasificados como materiales promisorios para la región. Con un comportamiento intermedio en la variable de producción acumulada estuvieron las selecciones de naranja 'Valencia Rhode Red', 'Delta' y 'Midknight'; mientras que la naranja 'Cara-Cara Navel', la toronja 'Río Red' y el tangelo 'Pear' se pueden descartar como potenciales, pues no se adaptaron a las condiciones ambientales del lugar de evaluación, presentando una baja producción y pobre calidad del fruto. El tangelo 'Pear' se confirmó como susceptible a Phytophthora en la evaluación realizada.

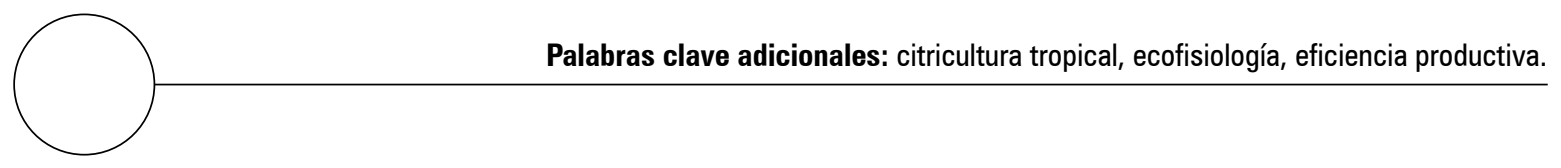

Centro de Investigación La Libertad, Corporación Colombiana de Investigación Agropecuaria (Corpoica), Villavicencio (Colombia).

2 Universidad de Ciencias Aplicadas y Ambientales (UDCA), Bogotá (Colombia).

3 Autor para correspondencia. jorduz@corpoica.org.co 


\section{ABSTRACT}

The performance of 13 commercial varieties and hybrids of citrus under conditions of the Piedmont Plains in the department of Meta (Colombia) was assessed in order to select potentially productive citrus cultivars with suitable fruit quality for consumption. The growth variables (plant height, crown diameter), productive potential (yield per plant and fruit weight) and fruit quality (total soluble solids, total titratable acidity and juice content) were measured. The cultivars that showed better productive potential and fruit quality were 'Dancy' tangerine, 'Sweet Crescent' orange, 'Star Ruby' grapefruit, 'Ellendale' tangor and 'Orlando' tangelo, which can be classified as promising materials for the region. 'Rhode Red Valencia', 'Delta' and 'Midknight' oranges had intermediate behavior in the cumulative output variable, while 'Cara-Cara Navel' orange, 'Río Red' grapefruit and 'Pear' tangelo can be ruled out as potential cultivars as they are not adapted to the environmental conditions of the area that was evaluated, with low production and poor fruit quality. According to the study, the 'Pear' tangelo was confirmed as being susceptible to Phytophthora.

Additional key words: tropical citrus production, ecophysiology, production efficiency.

Fecha de recepción: 08-07-2011

Aprobado para publicación: 21-11-2011

INTRODUCCIÓN

La producción de cítricos se obtiene en las regiones tropicales y subtropicales (excluyendo zonas con inviernos severos), situadas principalmente entre los $40^{\circ}$ de latitud norte y sur. En las regiones tropicales, ubicadas entre los $23,5^{\circ}$ de latitud norte y sur, los cítricos se cultivan desde 0 a $2.000 \mathrm{msnm}$. Dependiendo de dicha altitud, las regiones citrícolas tropicales se clasifican en: trópico alto (1.500 a $2.000 \mathrm{msnm})$, medio (800 a $1.500 \mathrm{msnm}$ ) y bajo (0 a $700 \mathrm{msnm}$ ) (OrduzRodríguez, 2007a). Colombia posee alrededor de 62.000 ha de cítricos, ubicadas en su mayoría en el trópico medio y bajo.

La citricultura colombiana posee un escaso número de variedades cultivadas lo cual ocasiona una excesiva concentración de la cosecha, por ejemplo: la mandarina 'Arrayana' (el material sembrado en el piedemonte llanero), tiene solo una cosecha de importancia comercial a final del año. La totalidad de la producción se recoge en 2 meses con el consiguiente deterioro del precio, situación que se repite en las otras especies de cítricos.
El crecimiento y desarrollo de los cítricos es afectado de forma directa por las condiciones ambientales presentes en una región determinada (Agustí, 2003); siendo para el agricultor muy importantes el rendimiento por planta y la calidad (interna y externa) del fruto, lo que condiciona el tipo de uso del fruto y los mercados adonde se dirigirá la producción. Las variedades de las principales especies cultivadas (naranja, mandarina, toronja y los híbridos de tangor y tangelo) han sido seleccionadas para las zonas entre los $20^{\circ} \mathrm{y}$ $40^{\circ}$ de latitud en ambos hemisferios (conocidas como los cinturones citrícolas). En estas condiciones climáticas, la inducción floral se presenta por la detención del crecimiento ocasionada por bajas temperaturas. La maduración del fruto también se presenta en esta época (invierno), lo que promueve la síntesis de carotenos en la piel de las frutas dando origen al color naranja intenso característico de los cítricos de estas regiones (Reuther, 1973; Young y Ericsson, 1961).

Los principales mercados de importación y de consumo exigen estas variedades por las carac- 
terísticas de calidad interna y externa del fruto. Sin embargo, cuando estas variedades se cultivan en condiciones tropicales presentan un comportamiento en producción y calidad diferentes al que tienen cuando se cultivan en condiciones del subtrópico. Por esta razón, es necesario evaluarlas en el trópico alto, bajo o medio, dependiendo de sus requerimientos de unidades de calor; lo cual tiene una alta correlación con variedades tempranas, intermedias y tardías (Orduz-Rodríguez, 2007a). Para el desarrollo de nuevas variedades recomendadas en condiciones tropicales, es necesario conocer su potencial de rendimiento durante varias cosechas y evaluar, por tanto, su comportamiento en producción por planta, y la calidad interna y externa de los frutos; además de conocer su susceptibilidad a plagas y enfermedades. Esta información es fundamental para el desarrollo de nuevas variedades para la citricultura tropical.

El piedemonte llanero está ubicado entre 300 y $400 \mathrm{msnm}$; siendo la temperatura media anual de $26^{\circ} \mathrm{C}$ (Ideam, 2010). En estas condiciones se presenta una alta acumulación de unidades de calor (UC, unos 5.000 para los cítricos) que comprenden temperaturas medias diarias superiores a $12,5^{\circ} \mathrm{C}$, la cual se considera el cero fisiológico para las plantas de cítricos (Davies y Albrigo, 1994; Mendel, 1969); con un promedio de 4.930 UC acumuladas para el Centro de Investigación (C.I.) La Libertad, ubicado en el piedemonte llanero de Colombia (Orduz-Rodríguez, 2007b). Las regiones tropicales bajas poseen hasta tres veces más UC acumuladas al año que las regiones citrícolas de clima mediterráneo, las cuales pueden oscilar entre 1.600-1.700, como es el caso de Valencia (España) y Riverside en California; o pueden ser entre 30 a $40 \%$ superiores a las regiones citrícolas del subtrópico que son de 3.700 (Orlando, FL), o 3.000 en Limeira (Sao Paulo, Brasil) (Mendel, 1969). La acumulación de UC se correlaciona fuertemente con el índice de crecimiento y calidad del fruto (siempre y cuando el agua y los nutrientes no sean limitantes), de tal manera que la mayor acumulación de UC au- menta la respiración de las plantas lo que disminuye el contenido de sólidos solubles y la acidez de los frutos (Davies y Albrigo, 1994).

La floración de los cítricos en condiciones tropicales y en regiones cerca al ecuador (climas isotérmicos), es inducida por la detección del crecimiento vegetativo en la época seca, que se inicia en noviembre y se prolonga hasta mediados del mes de marzo. Quince días después de finalizar el déficit hídrico por el inicio de la época lluviosa se presenta la floración, seguida de la caída de pétalos (Orduz-Rodríguez y Fischer, 2007); 5 semanas del final de la antesis la caída fisiológica de frutos en la mandarina 'Arrayana' ( $C$. reticulata Blanco) (Orduz-Rodríguez et al., 2009); posteriormente el fruto continúa su crecimiento y desarrollo, llegando a su madurez al final de la época lluviosa, entre 8,5 a 9,0 meses después de la floración para las variedades comerciales de mandarina 'Arrayana', tangelo 'Minneola' y la naranja 'Valencia'.

Teniendo en cuenta que las principales regiones citrícolas del mundo están ubicadas en el subtrópico y que las variedades más importantes (tanto para el mercado en fresco como para fruta transformada) están seleccionadas para estas regiones, es necesario evaluar el potencial de rendimiento y la calidad del fruto de cultivares con potencial en el mercado en las condiciones ambientales del trópico bajo del país. Esto con el propósito de seleccionar germoplasma que pueda continuar su periodo de evaluación para recomendar nuevas variedades a los productores del piedemonte llanero y otras zonas con características similares.

\section{MATERIALES Y MÉTODOS}

Se evaluaron 13 variedades e híbridos de cítricos provenientes de la Universidad de California, Riverside (tabla 1) e introducidos a Colombia por el Grupo de Virología Vegetal de la Universidad Nacional de Colombia, sede Bogotá. El material 
fue plantado en la finca Las Brisas de Cumaral (Meta), de las cuales se tomaron las yemas para injertar las plantas en el año 1999. El establecimiento de las plantas en campo se hizo en septiembre del año 2000, en un lote de la terraza alta del C.I. La Libertad de Corpoica en Villavicencio, departamento del Meta $\left(04^{\circ} 03^{\prime} \mathrm{N}, 73^{\circ} 29^{\prime} \mathrm{W}, 336\right.$ msnm), con una distancia de plantación de $8 \times$ $6 \mathrm{~m}$ y seis plantas por cultivar. Como patrón se utilizó el mandarino Cleopatra (C. reticulata) que es el más frecuente en la región y el más usado en la evaluación del germoplasma en los Llanos Orientales.

El manejo del cultivo se realizó siguiendo las recomendaciones de Orduz y Baquero (2003) para el piedemonte llanero. Esta zona presenta un régimen pluviométrico monomodal, en el cual la temporada húmeda se inicia en marzo y termina en noviembre. La precipitación promedio anual oscila entre 2.500 y $3.800 \mathrm{~mm}$ (en promedio $2.918 \mathrm{~mm}$ ), con una temperatura media de $26^{\circ} \mathrm{C}$ (Ideam, 2010). La clasificación climática de la región corresponde a bosque húmedo tropical (IGAC, 2004). El tipo de suelo del área experimental se clasifica como Typic Haplustox, de textura franco arenosa (FA). En la clasificación regional estos suelos se incluyen en la clase IV (terrazas altas), la cual es recomendada para cítricos en la zona (Orduz y Baquero, 2003).

A partir de 2003, cada año, al finalizar el ciclo de crecimiento anual (al terminar la época lluviosa, en el mes de diciembre), se evaluó la modificación del crecimiento de las plantas y el volumen de la copa que fue calculado usando la fórmula de Turrel (1946):

$$
V=0,5236 \times H \times D^{2}
$$

Dónde: $V=$ volumen de la copa, $H=$ altura de la copa y $D=$ diámetro de la copa.

Los árboles iniciaron producción al cuarto año (2004), a partir de entonces se llevó registro anual durante 5 años, de peso del fruto, número de frutos y producción por árbol. Los frutos maduros se cosecharon a partir de noviembre $(9$ meses después de la floración). En las variables de rendimiento anual y acumulado, se calculó el promedio por árbol de seis plantas en cada cosecha y se determinó el error típico para el pro-

Tabla 1. Variedades e híbridos de cítricos evaluados en el piedemonte llanero del departamento del Meta. C.I. La Libertad, Villavicencio. Establecimiento en el año 2000.

\begin{tabular}{|l|l|l|}
\hline N ${ }^{0}$ & \multicolumn{1}{|c|}{ Variedad } & \multicolumn{1}{|c|}{ Nombre científico } \\
\hline 1 & Naranja Rhode Red Valencia CRC 3995 LO48 (C3) AC & Citrus sinensis (L.) Osbeck \\
\hline 2 & Naranja Cara-Cara Navel CR 3994129 (E2) AC & C. sinensis (L.) Osbeck \\
\hline 3 & Naranja Delta Valencia CR 3963189 (C3) AC & C. sinensis (L.) Osbeck \\
\hline 4 & Tangor Ellendale VI 464 CA. 1-21-94 & C. sinensis (L.) Osbeck x C. reticulata Blanco \\
\hline 5 & Toronja Star Ruby CRC 3110-1-21-94 & C. paradisi Macf. \\
\hline 6 & Mandarina Dancy VI 386-1-21-94 & C. reticulata Blanco \\
\hline 7 & Tangelo Orlando CRC 3814-1-21-94 & C. reticulata Blanco x C. paradisi Macf. \\
\hline 8 & Tangelo Page CRC 3616 USDA-1-21-94 & C. reticulata Blanco x C. paradisi Macf. \\
\hline 9 & Tangelo Minneola CRC 3340 & C. reticulata Blanco x C. paradisi Macf. \\
\hline 10 & Tangelo Pear CRC 2849-1-21-94 & C. reticulata Blanco x C. paradisi Macf. \\
\hline 11 & Toronja Río Red CRC 3916 & C. paradisi Macf. \\
\hline 12 & Naranja Midknight Valencia CRC 3955544 (C3) AC & C. sinensis (L.) Osbeck \\
\hline 13 & Naranja Crescent Sweet CRC 3802 396 (FB) AC & C. sinensis (L.) Osbeck \\
\hline
\end{tabular}


medio acumulado. La eficiencia productiva se calculó dividiendo la producción por planta $(\mathrm{kg})$ en el volumen de copa calculado $\left(\mathrm{m}^{3}\right)$ y se determinó el error típico para el promedio de seis cosechas de nueve cultivares y de cinco cosechas para los cuatro restantes. Para las variables de calidad del fruto (peso, contenido de jugo, acidez total, grados Brix) se tomó una muestra de 10 frutos por cada árbol, seleccionados al azar en cada cosecha, que fueron pesados y a los cuales se les extrajo el jugo con un exprimidor industrial. El contenido de jugo fue medido con probeta graduada. La acidez total titulable (ATT) se determinó como equivalente del ácido cítrico por titulación con $\mathrm{NaOH} 0,1 \mathrm{~N}$ hasta $\mathrm{pH} 8,2$; los sólidos solubles totales (medidos con refractómetro manual) corregidos (SSTc) se determinaron a través de la siguiente fórmula:

$$
S S T c=0,194 \times A \times S S T
$$

Dónde: $A=$ porcentaje de acidez y $S S T={ }^{\circ}$ Brix.

La información sobre características de los frutos y de calidad fue promediada para todas las cosechas; se realizaron pruebas de dispersión y se presenta con el error típico entre cosechas.

\section{RESULTADOS Y DISCUSIÓN}

\section{Comportamiento vegetativo}

\section{Volumen de copa}

La última lectura realizada señala a las plantas de tangelo 'Orlando' y 'Minneola' como las de mayor tamaño de todos los evaluados, siendo para el primero $89,5 \mathrm{~m}^{3}$ y para el segundo 88 $\mathrm{m}^{3}$. Dentro del mismo grupo de plantas grandes estuvieron el tangor 'Ellendale', la mandarina 'Dancy', el tangelo 'Page' con valores de tamaño de copa ubicados entre 50 y $72 \mathrm{~m}^{3}$ (tabla 2). Estas plantas obtuvieron un gran tamaño de copa superando al obtenido por otros cultivares en otras latitudes; por ejemplo, la mandarina 'Ponkan' injertada en Cleopatra obtuvo 72,3 m³, 11 años después del transplante en condiciones de Parana (sur de Brasil); siendo el mayor tamaño de varios patrones evaluados (Roose et al., 1989).

Las naranjas 'Valencia Midknight' y 'Valencia Rhode Red' presentaron al final de la evaluación un tamaño ubicado en el tercio inferior de 21,3 y $33,4 \mathrm{~m}^{3}$, mientras que 'Valencia Delta' presentó un tamaño medio, siendo la mitad de las plantas de mayor tamaño (los tangelos 'Minnneola' y 'Orlando'). La 'Valencia Midknight' y la 'Delta' fueron evaluadas en condiciones de trópico seco de México utilizando riego suplementario obteniendo en el noveno año un volumen de copa superior a los $60 \mathrm{~m}^{3}$, superando el tamaño obtenido en la evaluación en el piedemonte llanero (Medina et al., 2007). Las diferencias pueden estar relacionadas con las condiciones de clima (mayores temperaturas en especial en el verano), suelos de mayor fertilidad y en el patrón utilizado; mientras que evaluaciones en Colima (México) con el clon regional de Valencia, injertadas sobre Cleopatra, reportan desarrollos de copa de $18,4 \mathrm{~m}^{3}$ en plantas de 8 años (Pérez-Zamora et al., 2002), este volumen es similar al obtenido con la naranja 'Valencia Midknight' (18,7 $\left.\mathrm{m}^{3}\right)$ en plantas con la misma edad; mientras que la naranja 'Valencia Rhode Red' presentó 40\% $\left(26,7 \mathrm{~m}^{3}\right)$ más de volumen de copa, siendo ambas superadas por la naranja 'Valencia Delta' en $89 \%\left(34,8 \mathrm{~m}^{3}\right)$. Estos resultados sugieren que además de las influencias ambientales y del patrón, cada genotipo así sea una selección de la misma variedad, presenta un comportamiento diferenciado, por lo cual siempre demanda su evaluación en las condiciones locales.

Las toronjas 'Río Red' y 'Star Ruby' al finalizar la evaluación presentaron un tamaño intermedio $\left(38,4\right.$ y $47,2 \mathrm{~m}^{3}$, respectivamente), lo que permitiría pensar en que se puede aumentar el número de plantas/ha y de esa forma aumentar los rendimientos. La mandarina 'Dancy' (65,3 $\mathrm{m}^{3}$ ) presentó el cuarto lugar en tamaño después de los tangelos y el del tangor 'Ellendale'. Los otros cultivares como 'Cara Cara Navel' y el tan- 
Tabla 2. Volumen de copa $\left(\mathrm{m}^{3}\right)$ de 13 cultivares de cítricos (sobre el patrón mandarina Cleopatra), plantados en el año 2000 en el C.I. La Libertad, Villavicencio (Meta).

\begin{tabular}{|c|c|c|c|c|c|c|c|}
\hline Cultivares/años & 2004 & 2005 & 2006 & 2007 & 2008 & & 2009 \\
\hline Tangelo Pear & $4,55 \mathrm{gh}$ & $7,61 \mathrm{~h}$ & $11,45 \mathrm{i}$ & $16,14 \mathrm{k}$ & 16,86 & & $17,58 \mathrm{k}$ \\
\hline Valencia Midknight & 3,65 hi & 5,61 i & $11,74 \mathrm{i}$ & $16,20 \mathrm{k}$ & 18,72 & j & $21,25 j$ \\
\hline Naranja Crescent Sweet & $3,58 \quad \mid$ & 6,13 i & $13,06 \mathrm{~h}$ & $17,91 \mathrm{j}$ & 25,27 & i & 32,63 i \\
\hline Valencia Rhode Red & $4,59 \mathrm{gh}$ & $7,73 \mathrm{~h}$ & $14,01 \mathrm{~g}$ & $19,98 \mathrm{i}$ & 26,68 & h & $33,38 \mathrm{i}$ \\
\hline Toronja Río Red & 4,34 ghi & $5,95 \mathrm{i}$ & 8,89 j & $13,12 \quad$ & 25,76 & ih & $38,41 \mathrm{~h}$ \\
\hline Cara-Cara Navel & $5,76 f$ & $9,53 \mathrm{~g}$ & $16,09 f$ & $26,51 \mathrm{~g}$ & 34,35 & $g$ & $42,18 \mathrm{~g}$ \\
\hline Valencia Delta & $5,19 \mathrm{gf}$ & $8,70 \mathrm{~g}$ & $18,05 \mathrm{e}$ & $25,17 \mathrm{~h}$ & 34,77 & $g$ & $44,36 \mathrm{f}$ \\
\hline Toronja Star Ruby & $16,42 \quad \mathrm{c}$ & $2609 \mathrm{c}$ & $27,92 d$ & $37,81 \mathrm{e}$ & 42,48 & $f$ & $47,15 \mathrm{e}$ \\
\hline Tangelo Page & $13,03 \mathrm{~d}$ & $21,78 \mathrm{~d}$ & $37,01 \quad b$ & $44,51 \quad c$ & 48,01 & e & 51,51 d \\
\hline Mandarina Dancy & $12,10 \mathrm{~d}$ & $18,22 \mathrm{e}$ & $34,48 \quad c$ & $35,22 f$ & 50,27 & $d$ & $65,32 \mathrm{c}$ \\
\hline Tangor Ellendale & $10,91 \mathrm{e}$ & $16,92 \mathrm{f}$ & $27,82 d$ & $42,84 d$ & 57,12 & $\mathrm{C}$ & $71,39 \quad b$ \\
\hline Tangelo Minneola & $17,63 \mathrm{~b}$ & $29,22 \quad b$ & $37,45 \mathrm{~b}$ & $53,74 \quad b$ & 70,86 & $b$ & $87,97 \quad a$ \\
\hline Tangelo Orlando & 23,56 a & 33,93 a & 44,53 a & $64,30 \quad a$ & 76,90 & $\mathrm{a}$ & $89,51 \quad a$ \\
\hline
\end{tabular}

Promedios con letras distintas, en la misma columna, indican diferencia significativa según la prueba de Tukey $(P \leq 0,05)$.

gelo 'Page' desarrollaron un tamaño intermedio, mientras que la naranja 'Crescent Sweet' presentó el tercer menor tamaño, aunque sin diferencias estadísticas con la 'Valencia Rhode Red'.

\section{Rendimiento y eficiencia productiva}

\section{Rendimiento anual y acumulado}

La producción se inició en 2004 para nueve de los trece materiales evaluados; aunque la cantidad obtenida no fue representativa comercialmente para ninguno de los cultivares (tabla 3). En 2005, solo la variedad de toronja 'Río Red' presentó una producción aceptable desde el punto de vista comercial con $80 \mathrm{~kg} / a ́ r b o l$, siendo el material más precoz de los evaluados. Este comportamiento generalizado (de tardía entrada en producción), puede estar relacionado con el uso del patrón mandarina Cleopatra que le transmite esta característica a las variedades que se injertan sobre él (Castle, 1987).

En las seis cosechas evaluadas la mayor producción acumulada la obtuvo la mandarina 'Dancy’ con 546,2 kg/árbol, seguida por los tangelos
'Orlando' y 'Page', con 430,8 y 330,6 kg/árbol, respectivamente. Estas producciones son sobresalientes, comparadas con los rendimientos de las mejores variedades de la evaluación de 26 genotipos de naranja durante cinco cosechas, en las mismas condiciones ambientales y de manejo. Las cuatro variedades que presentaron los mejores rendimientos acumulados de seis cosechas, estuvieron entre 114,1 a 145,3 kg/árbol (OrduzRodríguez y Avella, 2008). Estas producciones son significativamente menores que la obtenida para el material de naranja con mayor producción acumula para esta evaluación ('Cresecent Sweet', 293,9 kg/árbol) también en seis cosechas.

Por otro lado, el grupo de los materiales con menor producción acumulada estuvo conformado por tres naranjas: 'Cara-Cara Navel', 'Valencia Delta' y 'Valencia Midknight', con 104,2; 103,3 y $98,6 \mathrm{~kg} / a ́ r b o l$, respectivamente. Estas producciones son cinco veces menores que la obtenida por la mandarina 'Dancy'. 'Midknight' también presentó bajos rendimientos en evaluaciones realizadas en la región de Tecomán, Colima (México) (Medina et al., 2007); mientras que en la misma evaluación, 'Delta' estuvo en el grupo de 
los de mayor rendimiento, diferente a los resultados en la evaluación del piedemonte llanero. La diferencia en comportamiento puede deberse a las distintas condiciones ambientales de las dos localidades, como al patrón utilizado (naranja agrio Citrus aurantium) en México.

El deficiente comportamiento de la naranja 'CaraCara' puede explicarse por su condición de ser una variedad del grupo Navel que presenta una baja adaptación a condiciones de altas temperaturas acumuladas (Davies y Albrigo, 1994). Mientras que las bajas producciones obtenidas por el clon de tangelo 'Minneola' pueden deberse a que este material provenía de California, con 1.700 UC acumuladas anualmente (Mendel, 1969), ya que plantas del mismo hibrido seleccionadas de material existente en el piedemonte llanero, y evaluadas en las mismas condiciones experimentales han reportado producciones tres veces más altas para el mismo número de cosechas a pesar de que los cultivos están afectados por Psoriasis (Mateus et al., 2010). Es probable que la selección realizada en el país desde su ingreso en la década de 1960, haya permitido tener genotipos con mejor comportamiento en condiciones del trópico bajo, lo que señala la importancia del material comercial en actividades de limpieza sanitaria y programas de multiplicación de plantas para los citricultores.

\section{Eficiencia productiva}

De acuerdo con la tabla 4, la toronja 'Río Red' mostró el mejor promedio de eficiencia productiva, seguido por naranja 'Crescent Sweet' y de la mandarina 'Dancy'. Por otro lado, los materiales que presentaron la menor eficiencia fueron el tangelo 'Minneola', el tangor 'Ellendale' y la naranja 'Valencia Delta'.

Las naranjas 'Cara-Cara Navel' y 'Midknight', mostraron un comportamiento similar al reportado en México, con eficiencias de 1,4 y 1,1 respectivamente, mientras que la 'Valencia Delta' se comportó mejor en dichas condiciones, con eficiencia de 2,0 (Medina et al., 2007).

\section{Tabla 3. Rendimiento promedio anual y acumulado (kg/árbol) de seis cosechas en 13 cultivares de cítricos (sobre el patrón mandarina Cleopatra), plantados en el año 2000 en el C.I. La Libertad, Villavicencio (Meta).}

\begin{tabular}{|l|r|r|r|r|r|r|r|r|}
\hline \multicolumn{1}{|c|}{ Cultivares/años } & 2004 & 2005 & 2006 & 2007 & 2008 & 2009 & $\begin{array}{c}\text { Producción } \\
\text { acumulada } \\
\text { (kg/árbol) }\end{array}$ & $\begin{array}{c}\text { Error típico de } \\
\text { la producción } \\
\text { acumulada }\end{array}$ \\
\hline Valencia Midknight & 1,0 & 18,7 & 36,5 & 14,4 & 20,5 & 7,5 & 98,6 & $\pm 5,46$ \\
\hline Valencia Delta & & 12,8 & 26,4 & 8,8 & 44,8 & 10,5 & 103,3 & $\pm 7,15$ \\
\hline Cara-Cara Navel & 3,2 & 17,3 & 31,5 & 27,4 & 23,2 & 1,6 & 104,2 & $\pm 5,60$ \\
\hline Tangelo Minneola & & 1,8 & 10,0 & 37,6 & 49,1 & 28,5 & 127,0 & $\pm 9,05$ \\
\hline Toronja Río Red & 3,4 & 79,7 & 8,6 & 13,7 & 26,3 & 7,8 & 139,6 & $\pm 12,86$ \\
\hline Tangelo Pear & 14,9 & 3,7 & 57,4 & 17,0 & 43,3 & 10,0 & 146,4 & $\pm 9,44$ \\
\hline Tangor Ellendale & 3,2 & 4,8 & 5,5 & 6,2 & 121,6 & 50,0 & 191,2 & $\pm 21,25$ \\
\hline Valencia Rhode Red & & 3,7 & 32,2 & 110,4 & 31,1 & 18,2 & 195,6 & $\pm 18,06$ \\
\hline Naranja Crescent Sweet & & 3,3 & 19,3 & 115,1 & 137,4 & 18,8 & 293,9 & $\pm 27,18$ \\
\hline Toronja Star Ruby & 14,5 & 24,5 & 72,6 & 69,8 & 80,5 & 40,5 & 302,4 & $\pm 12,38$ \\
\hline Tangelo Page & 9,6 & 18,4 & 27,1 & 97,2 & 118,5 & 59,8 & 330,6 & $\pm 20,02$ \\
\hline Tangelo Orlando & 17,0 & 3,4 & 37,9 & 148,3 & 215,4 & 8,9 & 430,8 & $\pm 39,63$ \\
\hline Mandarina Dancy & 24,5 & 9,2 & 87,0 & 102,2 & 305,6 & 17,6 & 546,2 & $\pm 50,08$ \\
\hline
\end{tabular}


El error típico obtenido para la eficiencia productiva de los materiales, desde el año 2004 hasta el año 2009, es muy amplio en algunos casos; lo anterior está relacionado con la variabilidad anual de la producción, influenciada por los factores ambientales en especial con el déficit hídrico en la época seca y su incidencia en las fases de inducción floral, floración y cuajado. Deficiencias hídricas en las fases de floración-cuajado pueden afectar el rendimiento de la cosecha de esos años (Davies, 1997). Es probable que existan respuestas diferenciadas por genotipos, en los cuales los patrones tienen una fuerte influencia. La mayor eficiencia productiva la presentó la toronja 'Río Red' y se debe en gran parte a la cosecha obtenida en 2005 (tabla 3), cercana a los 80 kg/árbol, la cual afectó el valor final e influyó sobre el promedio obtenido por este cultivar $2,96 \mathrm{~kg} \mathrm{~m}^{-3}$, cercano al error típico de 2,26.

Un estudio publicado sobre mandarina 'Arrayana' en condiciones del piedemonte del Meta, mostró una eficiencia productiva de $3,4 \mathrm{~kg} \mathrm{~m}^{-3}$ con el mismo patrón (mandarina Cleopatra) en las primeras cinco cosechas, pero al variar el patrón a citrumelo Swingle la eficiencia aumentó a $8,0 \mathrm{~kg} \mathrm{~m}^{-3}$ (Orduz-Rodríguez y Fischer, 2007); teniendo en cuenta que el patrón Cleopatra está reportado como de tardía entrada en producción y disminuye el tamaño del fruto (Castle, 1987). Sería recomendable evaluar los materiales de mejor comportamiento con otros patrones con el fin de mejorar la precocidad, disminuir el tamaño de la planta y mejorar la eficiencia productiva.

Un gran volumen de copa no siempre se relaciona con una buena producción ni una óptima eficiencia productiva, como puede observarse en la tablas 3 y 4 (con el tangelo 'Minneola' y el tangor 'Ellendale', entre otros). En este ensayo las mayores eficiencias productivas las presentaron las plantas con producción media pero con un bajo volumen de copa, como es el caso de la naranja 'Crescent Sweet' y la toronja 'Río Red', este tipo de plantas pueden mejorar considerablemente la producción por hectárea al aumentar el número de plantas.

Tabla 4. Eficiencia productiva $\left(\mathrm{kg} \mathrm{m}^{-3}\right)$ en 13 cultivares de cítricos (sobre el patrón mandarina Cleopatra), plantados en el año 2000 en el C.I. La Libertad, Villavicencio (Meta).

\begin{tabular}{|l|c|c|c|c|c|c|c|}
\hline \multicolumn{1}{|c|}{ Cultivares/Años } & 2004 & 2005 & 2006 & 2007 & 2008 & 2009 & $\begin{array}{c}\text { Promedio con error } \\
\text { típico }\end{array}$ \\
\hline Tangelo Minneola & & 0,06 & 0,27 & 0,70 & 0,69 & 0,32 & $0,41 \pm 0,13$ \\
\hline Tangor Ellendale & 0,37 & 0,28 & 0,20 & 0,14 & 2,13 & 0,70 & $0,64 \pm 0,34$ \\
\hline Valencia Delta & & 1,47 & 1,46 & 0,35 & 1,29 & 0,24 & $0,96 \pm 0,30$ \\
\hline Cara-Cara Navel & 0,66 & 1,82 & 1,96 & 1,03 & 0,68 & 0,04 & $1,03 \pm 0,33$ \\
\hline Tangelo Orlando & 1,00 & 0,10 & 0,85 & 2,31 & 2,80 & 0,10 & $1,19 \pm 0,51$ \\
\hline Tangelo Page & 0,88 & 0,84 & 0,73 & 2,18 & 2,47 & 1,16 & $1,38 \pm 0,34$ \\
\hline Valencia Midknight & 0,36 & 3,34 & 3,11 & 0,89 & 1,09 & 0,35 & $1,52 \pm 0,60$ \\
\hline Toronja Star Ruby & 1,11 & 0,94 & 2,60 & 1,85 & 1,90 & 0,86 & $1,54 \pm 0,31$ \\
\hline Valencia Rodhe Red & & 0,48 & 2,30 & 5,53 & 1,16 & 0,55 & $2,00 \pm 0,92$ \\
\hline Tangelo Pear & 3,92 & 0,48 & 5,01 & 1,05 & 2,57 & 0,57 & $2,27 \pm 0,85$ \\
\hline Mandarina Dancy & 2,69 & 0,51 & 2,52 & 2,90 & 6,08 & 0,27 & $2,50 \pm 0,94$ \\
\hline Naranja Crescent Sweet & & 0,54 & 1,48 & 6,42 & 5,44 & 0,58 & $2,89 \pm 1,26$ \\
\hline Toronja Río Red & 1,15 & 13,40 & 0,97 & 1,05 & 1,02 & 0,20 & $2,96 \pm 2,29$ \\
\hline
\end{tabular}




\section{Calidad de los frutos}

La producción de cítricos en Colombia está destinada casi en su totalidad al mercado en fresco, en especial para su consumo como jugo en los hogares en el caso de las naranjas y el tangelo; y para consumo directo en el caso de la mandarina. En el proceso de selección en esta fase se hace énfasis en los aspectos cuantitativos de los frutos y en menor medida los aspectos cualitativos como el color externo.

\section{Peso fresco del fruto}

Los frutos de tangelo 'Minneola' y naranja 'Valencia Midknight' presentaron el mayor peso fresco de los 13 cultivares evaluados con un promedio de 309 g, seguidos de 'Cara-Cara Navel' con $292 \mathrm{~g}$ por fruto (figura 1). La baja producción de estas variedades permite un buen desarrollo del fruto. En el caso de naranja, los frutos de primera calidad se consideran por encima de $230 \mathrm{~g}$, por lo cual la naranja 'Crescent Sweet' con 200 g se clasificaría como fruto de tamaño pequeño y con altas probabilidades de tener problemas de mercadeo, mientras que la naranja 'Valencia Delta' tendría el mejor tamaño para el merado. En una evaluación de 26 cultivares de naranja en el piedemonte llanero del Meta, los frutos de naranja 'Valencia' regionales obtuvieron pesos entre 235 y 265 g que pueden ser el estándar para los mercados de fruta fresca en el país (OrduzRodríguez y Avella, 2008).

El tamaño del tangelo 'Orlando' (240 g por fruto), unido a la forma redonda de fruto, limita el interés comercial de este cultivar debido a su parecido con los frutos de naranja; en consecuencia, los productores reciben el mismo precio/kg, siendo menor que lo recibido por los frutos del

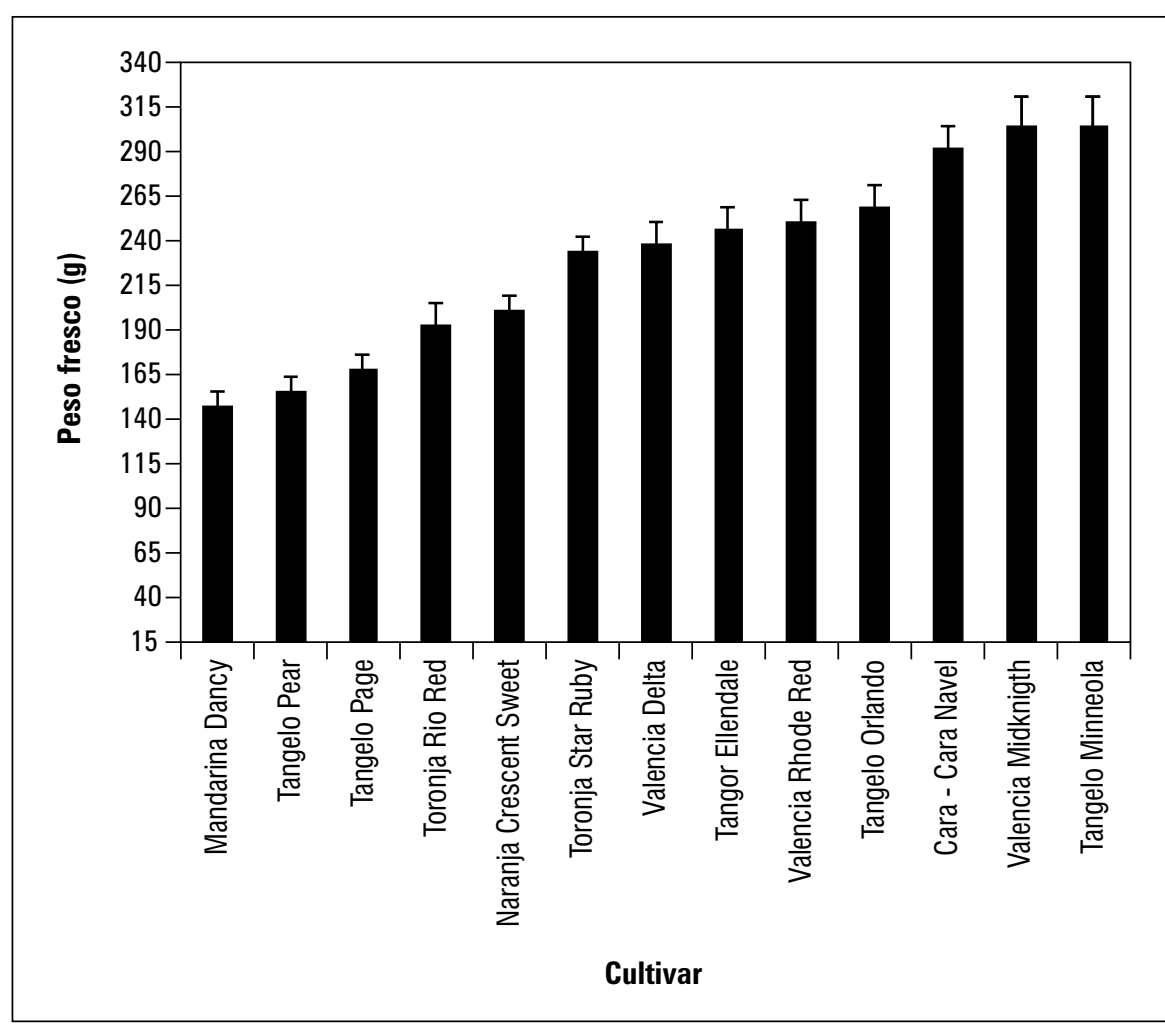

Figura 1. Peso fresco de frutos en 13 cultivares de cítricos (sobre el patrón mandarina Cleopatra) durante cinco cosechas. Árboles plantados en el año 2000 en el C.I. La Libertad, Villavicencio (Meta). Las barras sobre las columnas indican el error típico. 
tangelo 'Minneola'. El fruto de 'Minneola' es el de mayor circulación en el mercado nacional (Mateus et al., 2010).

El bajo peso del tangelo 'Page' (168 g), sumado a su bajo rendimiento y problemas sanitarios (Phytophthora), presentados en campo, lo eliminan como cultivar de interés para la región. En el caso de la mandarina 'Dancy', el bajo peso del fruto (150 g), puede estar relacionado con el patrón al que fue injertado (como ya se mencionó con anterioridad), puesto que la variedad regional Arrayana, en las mismas condiciones y con el mismo patrón obtuvo pesos de 159 g, pero llegó a pesar $184 \mathrm{~g}$ sobre el citrumelo Swingle (OrduzRodríguez et al., 2009). Para el desarrollo de la mandarina 'Dancy' sería conveniente evaluarla injertada en otros patrones para mejorar el tamaño del fruto, factor importante en el mercado nacional de esta variedad.

\section{Contenido de jugo}

El menor contenido de jugo de los frutos usados para la elaboración de jugo casero (naranjas y tangor o tangelos), se considera del $40 \%$, privilegiando las variedades que estén por encima de esa cifra. De lo anterior se puede concluir que cinco materiales superaron este porcentaje, entre ellos, el tangelo 'Orlando' obtuvo el valor más alto con $52 \%$. Seis materiales se encuentran entre $33 \%$ y $39 \%$, y por último la naranja 'Cara-Cara Navel' y la toronja 'Star Ruby', con los menores valores (figura 2). Aunque en el caso de las Navel son variedades recomendadas para el consumo en fresco y no para su uso como jugo casero.

En similares condiciones de evaluación, los clones regionales de Valencia obtuvieron valores entre 45,6 a 49,1\% (Orduz-Rodríguez y Avella,

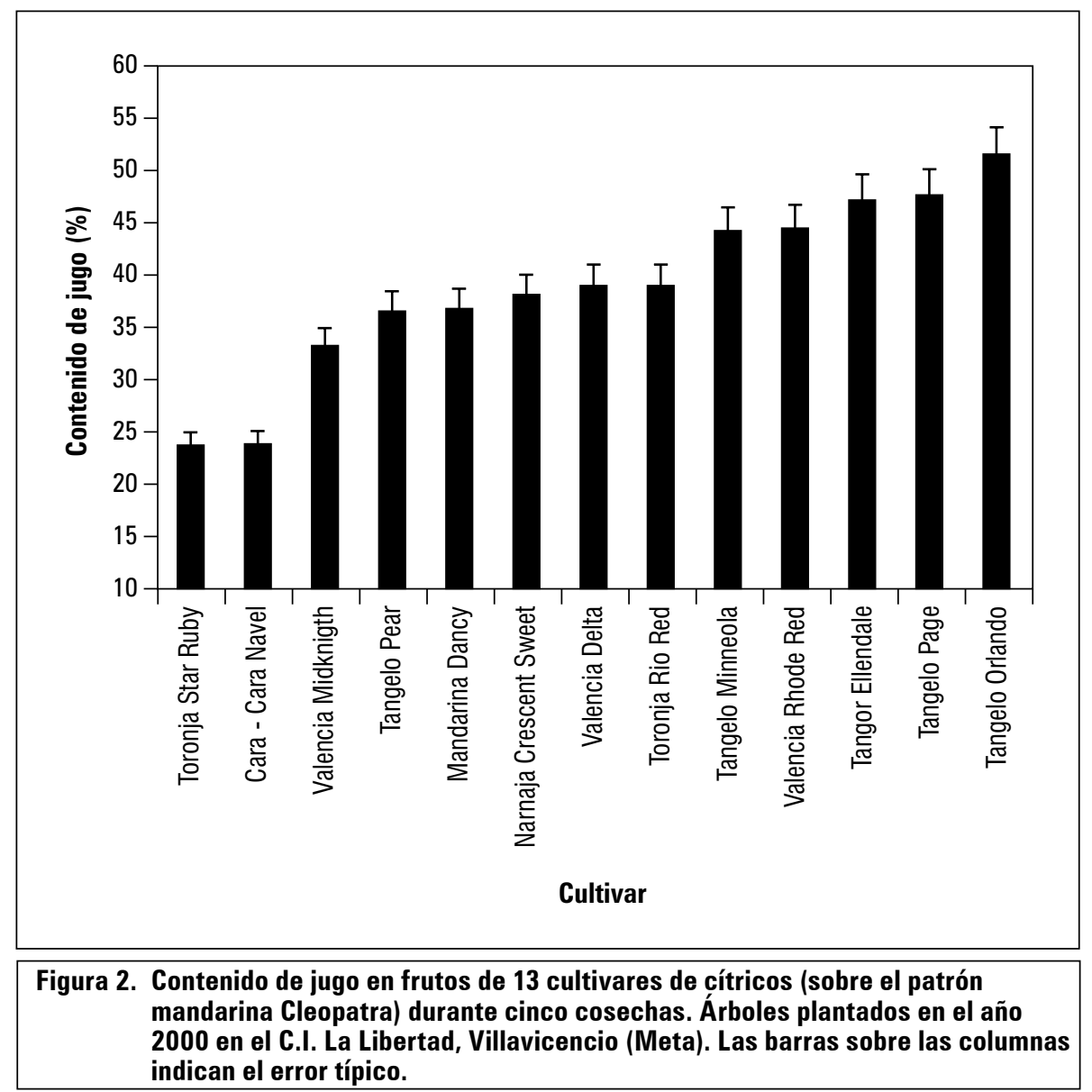

REV. COLOMB. CIENC. HORTIC. 
2008), lo que pone de manifiesto los bajos resultados de las naranjas; la única variedad por encima de este valor fue la 'Valencia Rhode Red'. La mandarina 'Dancy' obtuvo 38\% de jugo, cifra apropiada para este fruto, teniendo en cuenta que la mandarina 'Arrayana', injertada sobre el mismo patrón, desarrolló 39\% de contenido de jugo (Orduz-Rodríguez et al., 2009); mientras que para 'Ponkan', la mandarina más cultivada en Brasil, se reportó apenas $27,4 \%$ en promedio (Stenzel et al., 2003).

La toronja 'Río Red' presentó contenidos adecuados de jugo para este tipo de frutos, en cambio fue muy bajo para la toronja 'Star Ruby' con apenas $23 \%$. Los contenidos de jugo y las bajas producciones de la naranja 'Cara-Cara Navel', señalan su escasa adaptación a las condiciones ambientales del sitio de evaluación.

\section{Sólidos solubles totales (SST)}

La alta acumulación de unidades de calor en condiciones del piedemonte llanero del Meta aumenta la respiración de las plantas ocasionando un gasto energético que disminuye los SST y la acidez total titulable en los frutos. Por esta razón, todas las variedades e híbridos evaluados presentaron SST considerados como bajos, comparados con las regiones del trópico medio como Palmira o en zonas húmedas con inviernos cálidos como Florida (Estados Unidos) o Sao Paulo (Brasil) (Davies y Albrigo, 1994). El tangelo 'Page' y la naranja 'Crescent Sweet' fueron el único material que obtuvo concentraciones de SST superiores a 10 (figura 3), lo que indica una muy buena condición para su consumo en fresco o con posibilidades en la agroindustria. Por debajo de 8 estuvieron las toronjas 'Río Red' y ‘Star Ruby', y la 'Rhode Red Valencia'.

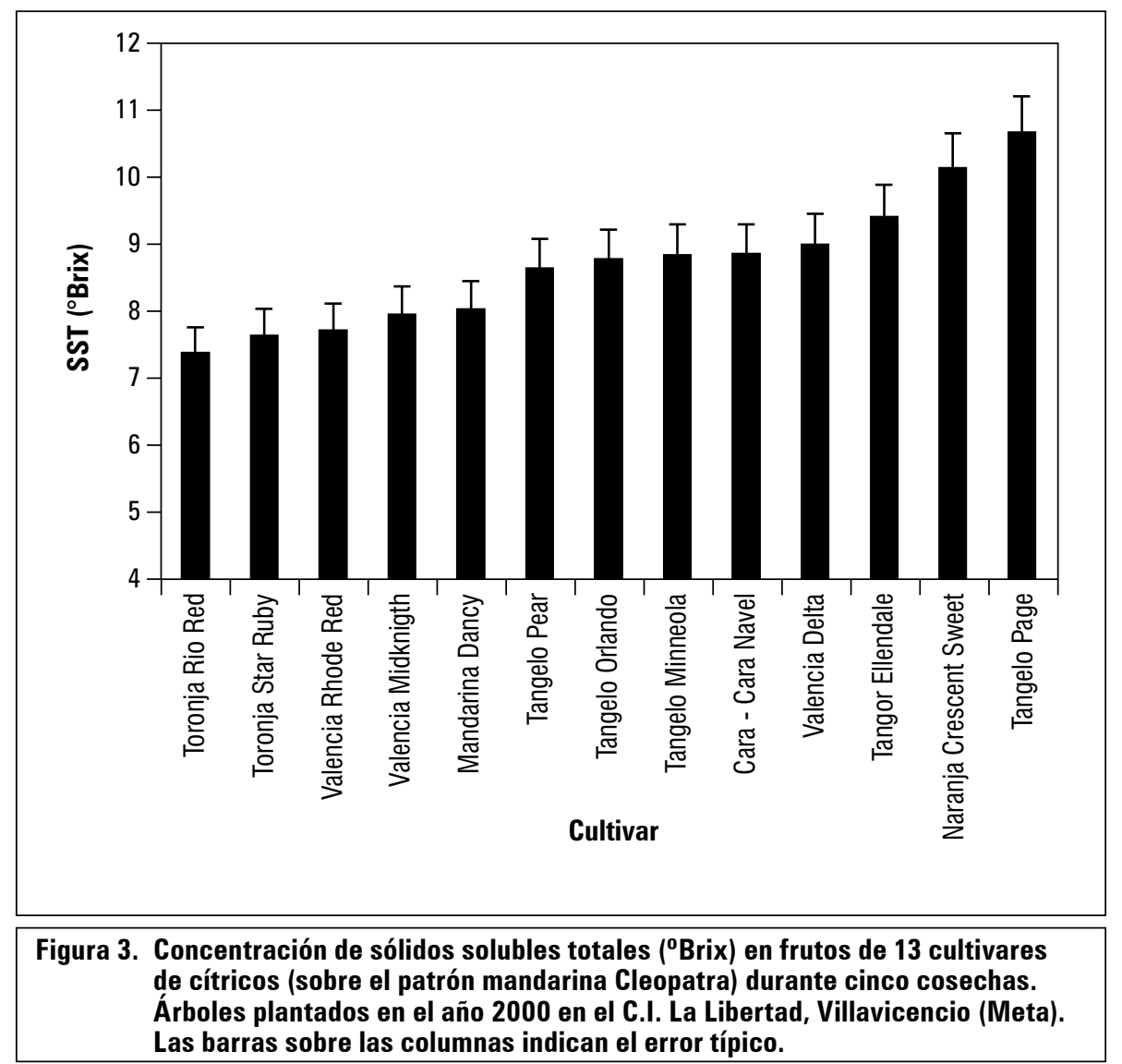

Vol. 5 - No. 2 - 2011 
La anterior información confirma la concentración moderada de SST al comparar los datos obtenidos de los 26 cultivares de naranja evaluados en el piedemonte llanero del Meta y que en su mayoría estuvieron entre 8,5 y 10,8 (Orduz-Rodríguez y Avella, 2008). Por otro lado, en Colima (México a $18^{\circ} \mathrm{N}$ ), la 'Valencia Delta' alcanzó 8,8\%, la 'Crescent Sweet' 10,5, la 'Midknight' 7,5 y la 'Cara-Cara' 8,9 'Brix (Medina et al., 2007), estos valores son un poco superiores a los obtenidos en el ensayo, hecho que puede estar influenciado por las menores temperaturas medias en la época de cosecha (invierno en el hemisferio norte), y por tanto tasas más bajas de respiración de las plantas que las del trópico bajo. En el caso de la mandarina 'Dancy' la concentración de SST (8\%) está en el rango obtenido con la 'Arrayana', que varió entre 7,2 con el patrón Volkameriana, a 8,2 con citrumelo Swingle (Orduz-Rodríguez et al., 2006), y 8,13 sobre mandarina Cleopatra (Orduz-Rodríguez et al., 2009). Sin embargo, estos contenidos siguen siendo moderados al compararse con los frutos de latitudes altas como el mediterráneo español (a $39,5^{\circ} \mathrm{N}$ ), en el cual por ejemplo 'Fortune' obtuvo 15,73 ${ }^{\circ}$ Brix (Salvador et al., 2003).

\section{Acidez total titulable (ATT)}

En la obtención de frutos de buena calidad para el consumo es más importante el descenso de la ATT que la concentración de los SST. El contenido de la acidez está influido por la temperatura (acumulación de UC) (Davies y Albrigo, 1994), la respuesta del genotipo evaluado a condiciones específicas y el patrón utilizado (Orduz y Avella, 2008). El descenso en la acidez se ha atribuido a la rápida respiración de ácidos orgánicos la cual incrementa al aumentar las temperaturas medias (Reuther y Ríos-Castaño, 1969). Los mismos autores señalaron que cuanto más alto es el régimen térmico día/noche, más baja es la concentración de ácidos; también reportaron los menores niveles de acidez en el trópico bajo para naranjas dulces comparado con la concentración obtenida en el trópico medio y las regiones subtropi- cales de California (Reuther y Ríos-Castaño, 1969). La información anterior explica por qué las menores concentraciones de ATT la obtienen los frutos de las naranjas 'Cara-Cara Navel' y la 'Crescent Sweet' (alrededor del 0,5\%) (figura $3 \mathrm{~A})$. En estas concentraciones, el jugo de la fruta se considera insípido; en el caso de la 'Crescent Sweet' este valor señala que tiene una madurez temprana o intermedia ( 6 a 8 meses después de caída de pétalos). Los frutos de tangelo 'Minneola', 'Page' y 'Orlando', la mandarina 'Dancy', el tangor 'Ellendale' y las naranjas 'Valencia Delta', 'Midknight' $y$ 'Rhode Red' obtuvieron entre 0,82 y $1,0 \%$ de acidez (figura $4 \mathrm{~A}$ ).

Los resultados de las naranjas Valencia del presente ensayo son cercanos a los obtenidos con clones regionales de Valencia evaluados por Orduz-Rodríguez y Avella (2008), los cuales presentaron valores de 0,9 a 1,0\% de ATT a los 9 meses después de antesis; mientras que en Colima (México), la naranja 'Crescent' alcanzó 0,59; la 'Delta' 1,20, y la 'Valencia Midknight', 0,9. Estos valores ligeramente más altos coinciden con lo planteado acerca de la influencia de las mayores temperaturas medias en la respiración de los ácidos (Davies, 1997), superiores en el piedemonte llanero, comparados con las temperaturas medias en México en el invierno del hemisferio norte. Los valores más altos los obtuvieron el tangelo 'Pear' y las toronjas 'Río Red' y 'Star Ruby' con 1,26; 1,70 y 1,93\%, respectivamente. Si bien, las toronjas no poseen amplia información en el trópico, los estudios de Reuther y Ríos-Castaño (1969) señalan que estas en condiciones del trópico bajo presentan baja acidez y buena calidad interna.

El índice de madurez (SST/ATT) es el principal factor relacionado con el sabor de las frutas y está vinculado a los gustos de las personas de las diferentes regiones (Orduz-Rodríguez et al., 2009). Este índice es diferencial para cada tipo del fruto. En el caso de las toronjas 'Río Red' y 'Star Ruby'; aunque los frutos presentan los índices más bajos; estos corresponden a una buena calidad de fruta para esta especie; corroborando 


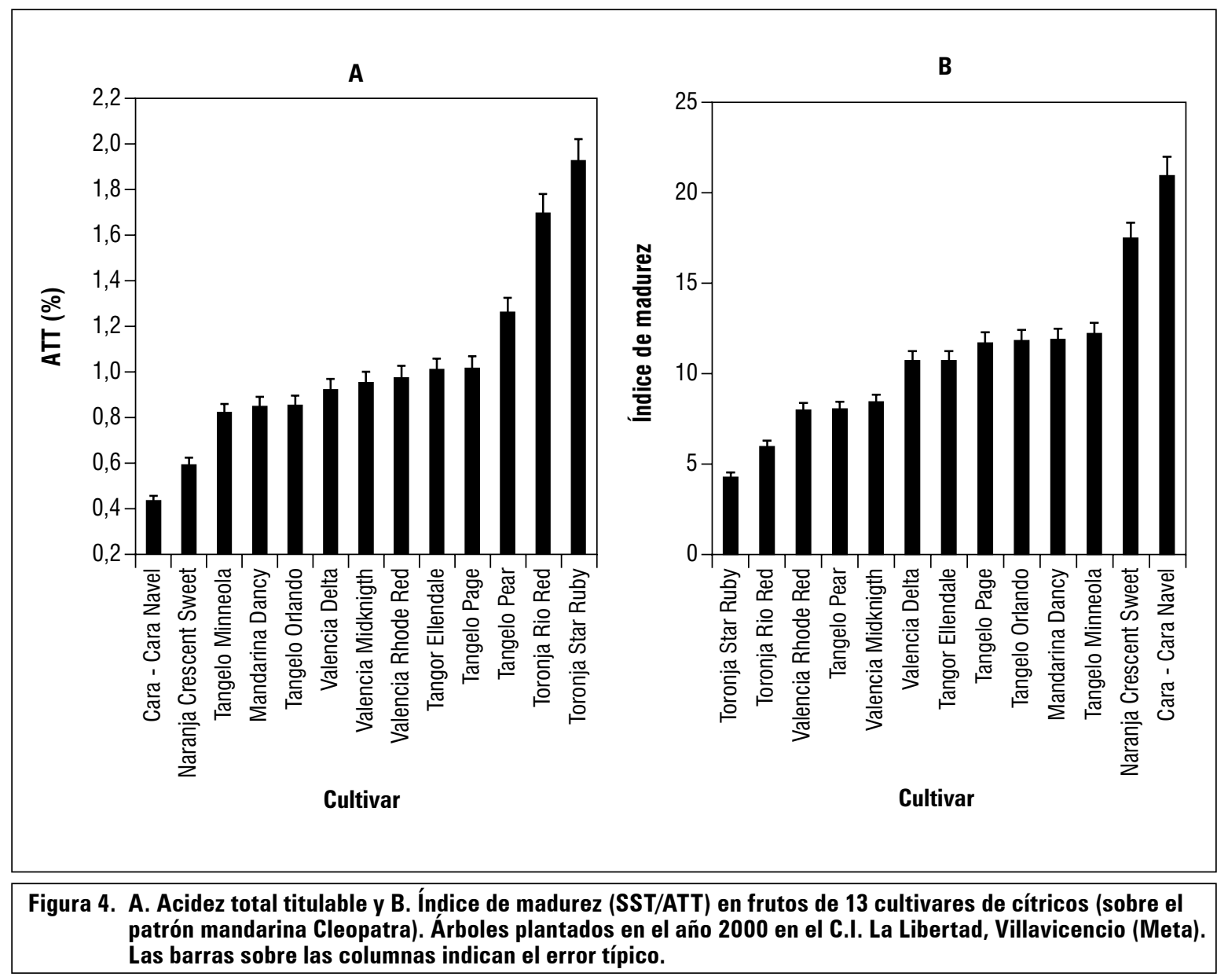

lo planteado por Davies y Albrigo (1994) para las toronjas en el trópico bajo. Es de destacar los valores intermedios que obtuvieron las naranjas valencianas 'Rhode Red' y 'Midknight' que estuvieron entre 8,0 y 8,4 (figura 4B) señalando que pueden ser cosechadas algún tiempo después de la fecha de muestreo (un índice de madurez de 9 es apto para consumo en naranja, aunque se considera que como los mejores valores están entre 10 y 12). Este comportamiento puede ser de interés para seleccionar cultivares con maduración tardía, lo que permitiría alargar la temporada de cosecha. Otro fruto que estuvo en ese rango fue el tangelo 'Pear'. Los demás cultivares evaluados estuvieron por encima de índice de madurez de 10, como es el caso de la naranja 'Valencia Delta', los tangelos 'Page', 'Orlando' y 'Minneola', el tangor 'Ellendale' y la mandarina 'Dancy', con valores entre 10 y 12. Estos valores señalan que los frutos pueden ser cosechados algún tiempo antes de los 9 meses después del final de antesis.

Las naranjas 'Crescent Sweet' y 'Cara-Cara Navel' presentaron los mayores valores de índice de madurez entre 17 y 20 , lo que señala que son frutas de madurez temprana (su cosecha puede hacerse alrededor de 6 a 7 meses después de la caída de pétalos). Esta característica junto con las producciones obtenidas y la calidad interna indican que 'Crescent Sweet' puede ser un material promisorio como fruto de cosecha temprana para el trópico bajo; mientras que 'Cara-Cara Navel', a pesar de su madurez temprana, no presenta ninguna de estas características, señalando que no posee buena adaptación a las condiciones ambientales de la región. El limitante que puede 
obtener la naranja 'Crescent Sweet' es el escaso tamaño del fruto lo que es una severa limitante para el mercado en fresco.

\section{CONCLUSIONES}

Los cultivares que presentaron un mejor comportamiento de las variables de rendimiento y calidad en las condiciones ambientales del trópico bajo, fueron la mandarina 'Dancy', naranja 'Crescent Sweet', toronja 'Star Ruby', el tangor 'Ellendale' y el tangelo 'Orlando'. Estos materiales deben ser considerados como potenciales para continuar su evaluación con el fin de que en el mediano plazo algunas puedan ser recomendadas a los productores de la región y de otras regiones del país de condiciones similares. Aunque en las evaluaciones posteriores se debe incorporar las exigencias de los mercados para cada cultivar evaluado.

Las selecciones de naranjas Valencia como 'Rhode Red', 'Delta' y 'Midknight' que presentaron un comportamiento intermedio y bajo potencial productivo, deben ser evaluadas en otros patrones teniendo en cuenta que los clones regionales de naranja 'Valencia' son los de mejor comportamiento en condiciones del trópico bajo.

Por su comportamiento deficiente en las principales variables evaluadas se deben descartar los cultivares de naranja 'Cara-Cara Navel', la toronja 'Río Red', el clon evaluado de tangelo 'Minneola' y el tangelo 'Pear'.

\section{AGRADECIMIENTOS}

Los autores expresan sus agradecimientos a: Mauricio Herrera, citricultor líder de la región y quien facilitó el germoplasma para ser evaluado en el C.I. La Libertad, a los directivos y al personal administrativo del C.I. La Libertad; Melba Mora, Capitolino Ciprian, Alfredo Pardo y David López por el acompañamiento y apoyo durante el desarrollo del proyecto; a la Ing. Agr. Diana Mateus y al Dr. Takumasa Kondo por su colaboración en las correcciones del texto final.

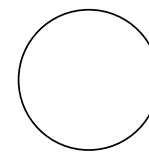

Agusti, M. 2003. Prólogo. En: Tadeo, F., J.L. Moya, D. Iglesias, M. Talón y E. Primo-Millo. E. (eds.). Histología y citología de cítricos. Generalitat Valenciana, Valencia. España.

Castle, W.S. 1987. Citrus rootstocks. pp. 361-369. En: Rom. R.C. y R.C. Carlson (eds.). Rootstocks for fruit crops. John Wiley and Sons, New York, NY.

Davies, F.S. 1997. An overview of climatic effects on citrus flowering and fruit quality in various parts of the world. pp. 1-4. En: Proc. Citrus Flowering and Fruit Short Course. IFAS, Citrus Research and Education Center, University of Florida, Lake Alfred, FL.
Davies, F.S. y L.G. Albrigo. 1994. Cítricos. Editorial Acribia, Zaragoza, España.

Ideam. 2010. Registros climáticos de la estación del C.I. La Libertad. Villavicencio, Colombia.

IGAC. 2004. Estudio general de suelos y zonificación de tierras. Departamento del Meta. IGAC, Bogotá.

Mateus, D., X. Pulido, A. Gutiérrez y J. Orduz. 2010. Evaluación económica de la producción de cítricos cultivados en el piedemonte del departamento del Meta durante 12 años. Orinoquia 14(1), 87-99.

Medina, V.M., G. Zapian, M. Robles, O. Pérez, M. Orozco, T. Williams y S. Becerra. 2007. Fenología y 
eficiencia productiva y calidad de la fruta de cultivares de naranjo en el trópico seco mexicano. Rev. Fitotec. Mex. 30(2), 133-143.

Mendel, K. 1969. The influence of temperature and light on the vegetative development of citrus trees. Proc. First Intl. Citrus Symp. 1, 259-265.

Orduz-Rodríguez. J.O. 2007a. Estudios ecofisiológicos y caracterización morfológica y molecular de la mandarina 'Arrayana' (Citrus reticulata Blanco) en el piedemonte llanero de Colombia. Tesis doctoral. Facultad de Agronomía, Universidad Nacional de Colombia, Bogotá.

Orduz-Rodríguez, J.O. 2007b. Ecofisología de los cítricos en el trópico: revisión y perspectivas. pp. 6776. En: Memorias Segundo Congreso Colombiano de Horticultura. Sociedad Colombiana de Ciencias Hortícolas, Bogotá.

Orduz-Rodríguez, J.O. 2008. Capítulo 2: Perspectivas y posibilidades del cultivo de cítricos en el Departamento de Casanare. pp. 8-13. En: Jaramillo, C. (ed.). Características de la citricultura del departamento del Casanare y recomendaciones para su mejoramiento productivo. Corpocia C.I. La Libertad. Produmedios, Bogotá.

Orduz-Rodríguez, J.O. y F. Avella. 2008. Comportamiento de 26 cultivares de naranja en condiciones del piedemonte del Meta. Rev. Colomb. Cienc. Hortíc 2(2), 157-172.

Orduz-Rodríguez, J.O. y J. Baquero. 2003. Aspectos básicos para el cultivo de los cítricos en el piedemonte llanero. Revista Achagua 7, 7-20.

Orduz-Rodríguez, J.O. y G. Fischer. 2007. Balance hídrico e influencia del estrés hídrico en la inducción y desarrollo floral de la mandarina 'Arrayana' en el piedemonte llanero de Colombia. Agron. Colomb. 25(2), 255-263.
Orduz-Rodríguez, J.O., H. Monroy, G. Fischer y A. Herrera A. 2009. Crecimiento y desarrollo del fruto de mandarina (Citrus reticulata) 'Arrayana' en condiciones del piedemonte del Meta. Colombia. Rev. Colomb. Cienc. Hortíc. 3(2), 149-160.

Pérez-Zamora, O., V. Medina y S. Becerra. 2002. Crecimiento y rendimiento de naranja Valencia injertada en 16 portainjertos de cítricos establecidos en suelo calcimórfico y calidad del jugo. Agrociencia 36, 137-148.

Reuther, W. 1973. Climate and citrus behaviour. pp. 280-337. En: Reuther, W. (ed.). The citrus industry. Vol. III. University of California, Davis, CA.

Reuther, W. y D. Ríos-Castaño. 1969. Comparison of growth, maturation and composition of citrus fruit in subtropical California and tropical Colombia. Proc. First Intl. Citrus Symp. 3, 277-300.

Roose, M.L., D.A. Cole, D. Atkin y R.S. Kupper. 1989. Yield and tree size of four Citrus cultivars on 21 rootstocks in California. J. Amer. Soc. Hort. Sci. 114(4), 678-684.

Salvador. A., J. Cuquerella y A. Monteverde. 2003. Efecto del quitosano aplicado como recubrimiento en mandarinas 'Fortune'. Rev. Iber. Tecnología Postcosecha 5(2), 122-127.

Stenzel, N., C. Neves, J. Gomes y C. Medina. 2003. Performance of Ponkan mandarin on seven rootstocks in Southern Brazil. HortScience 38(2), 176-178.

Turrel, F.M. 1946. Tables of surfaces and volumes of spheres and of prolates and oblates spheroides and spheroidal coefficients. University of California Press, Berkeley, CA.

Young, L.B. y L.C. Erickson. 1961. Influence of temperature on color change in Valencia oranges. Proc. Amer. Soc. Hort. Sci. 78, 197-200. 